\title{
Comportement des sols compactés : apports de la mécanique des sols non saturés
}

\begin{abstract}
P. DELAGE
École nationale des ponts et chaussées CERMES, Paris

6-8, avenue Blaise-Pascal 77455 Marne-la-Vallée

Cedex 2 delage@cermes.enpc.fr.
\end{abstract}

\section{J.-J. FRY}

Électricité de France Centre d'ingénierie hydraulique,

73373 Le Bourget-du-Lac Cedex
Les acquis de la mécanique des sols non saturés ne sont pas encore couramment utilisés dans la pratique de la conception et de la construction des ouvrages en terre (remblais, barrages), alors qu'ils fournissent des compléments d'information permettant une meilleure compréhension des phénomènes complexes intervenant dans ces ouvrages. On décrit ici le comportement des sols compactés sous cet angle, en abordant le rôle de la succion, la microstructure, le comportement en compression volumique et les propriétés de résistance au cisaillement. Ces données sont à compléter par des investigations expérimentales supplémentaires, mais certaines tendances existent et permettent d'éclairer le comportement d'un matériau finalement assez mal connu. Elles permettent également de proposer des modélisations simplifiées et efficaces des ouvrages en sol compacté, qui feront l'objet d'un autre article.

Mots-clés : sol compacté, remblai, barrage, succion, microstructure, comportement mécanique, ouvrages en terre,
NDLE: Les discussions sur cet article sont acceptées jusqu'au $1^{\text {er juillet } 2001 .}$
The mechanics of unsaturated soils is not systematically used in practice in the design and construction of earthworks (Embankments, dams). However, the mechanics of unsaturated soils can now provide some complementary information and allow a better understanding of the complex phenomena involved in earthworks. The paper describes the behaviour of cornpacted soils under the light of the mechanics of unsaturated soils, and deals with suction, microstructure, volumetric behaviour and shear' strength properties. Existing data should be completed by further experimental investigations. However, some trends exist, improving the understanding of the behaviour of a rather unknown material. Moreover, some simple and efficient models of compacted structures can be derived. which will be described in another paper.

Key words : compacted soil, embankment, dam, suction, microstructure, mechanical behaviour, earthworks. 


\section{Introduction}

Les développements apparus ces dernières années en mécanique des sols non saturés ${ }^{(1)}$, sur le plan de l'analyse expérimentale de la physique des phénomènes et celui de la modélisation du comportement, permettent une compréhension améliorée du comportement des ouvrages en terre, dont l'étude et le dimensionnement dans la pratique n'ont pas toujours pris en compte les spécificités dues à l'aspect non saturé. La différence essentielle engendrée par la présence d'une phase air dans les sols non saturés est reliée aux difficultés rencontrées dans les diverses tentatives d'extension de la contrainte effective de Terzaghi au cas non saturé. L'illustration la plus manifeste en est le phénomène d'effondrement, également appelé affaissement, qui correspond à la diminution de volume d'un sol non saturé non gonflant soumis à un remouillage sous charge. Les diverses expressions proposées de contrainte effective étendue aux sols non saturés (dont celle de Bishop 1959, cf. Annexe) sont proportionnelles à la succion et, comme elles décroissent lors d'un remouillage, elles prédisent un gonflement, incompatible avec la réduction de volume observée lors d'un effondrement. Ainsi, l'utilisation exclusive d'une contrainte de type Bishop est insuffisante pour une description complète du comportement des sols non saturés, et il est nécessaire d'y associer une autre variable de contrainte, dans le cadre bien admis de l'approche en variables indépendantes (voir Gens, 1995, pour la description des divers couples de variables de contraintes utilisés).

Cette différence fondamentale avec les sols saturés s'illustre également par le fait que, lors d'un chargement, il n'y a pas de transfert additif de contrainte totale entre l'eau, supposée incompressible, et les particules du sol, car l'eau n'emplit pas l'intégralité des vides existant (voir Fig. 1). Les vides emplis d'air permettent une réorganisation interne des grains et des ménisques existant aux contacts inter-particules, dont les conséquences macroscopiques en termes de volume ne sont pas descriptibles en termes de contrainte unique, dite effective. L'autre caractéristique importante des sols non saturés est la grande sensibilité de leurs propriétés mécaniques et hydrauliques aux variations de teneur en eau et/ou de decré de saturation.

On présente dans ce travail une description générale de la façon dont les travaux récents dans le domaine de la mécanique des sols non saturés permettent une appréhension plus complète du comportement des sols compactés qui constituent les ouvrages en terre (barrages en terre, remblais compactés). Ces éléments, essentiellement issus d'investigations de laboratoire sur des éprouvettes de taille décimétrique, ne répondent qu'en partie aux problèmes complexes soulevés par le comportement des ouvrages en remblai, dont beaucoup restent actuellement sans réponse. Il reste en particulier de nombreux efforts à développer pour mieux appréhender, au niveau du comportement global d'un massif compacté, les effets dus aux inévitables hétérogénéités mécaniques et hydrauliques intervenant lors de la construction, qui se traduisent, entre autres, par des variations de densité et de teneur en eau. Les

14 Une présentation de la mécanique non saturée est donnée dans Delage et Cui (2000 a et b). ouvrages construits font quelques dizaines de mètres de hauteur et parfois des kilomètres de longueur, ils sont réalisés à partir d'un matériau naturel provenant de zones d'emprunt elies-mêmes hétérogènes par nature, et sont compactés dans des conditions pas toujours contrôlées en fonction d'impératifs économiques contraignants. L'étude du changement d'échelle entre les éprouvettes de laboratoire et la dimension de ces ouvrages nécessite encore de nombreux efforts, qui devraient inclure en particulier la réalisation d'essais mécaniques de grande dimension, et l'étude d'ouvrages instrumentés sur le long terme (Mieussens, 2000). Malgré ces réserves, il semble que les travaux récents de rhéologie des sols non saturés puissent constituer un apport intéressant pour une meilleure compréhension du comportement des ouvrages en terre, en termes hydraulique et mécanique. Cet article présente le comportement des sols compactés vu à la lumière de la mécanique des sols non saturés. Il sera suivi d'un autre travail montrant comment ces concepts peuvent être intégrés dans un cadre de modélisation.

\section{2}

\section{Caractéristiques de compactage des sols}

Les problèmes relatifs au comportement des sols compactés, et leur positionnement par rapport aux sols fins saturés peuvent être commentés à partir du diagramme de compactage de la figure 2. La situation des sols fins saturés est décrite par l'hyperbole de saturation, qui est une relation biunivoque entre le poids volumique sec $\gamma_{d}$ et la teneur en eau $w$ :

$$
\gamma_{d}=\frac{\gamma_{s}}{1+\frac{w \gamma_{s}}{\gamma_{w}}}
$$

où $\gamma_{\text {s }}$ et $\gamma_{\text {w }}$ sont les poids volumiques des solides et de l'eau.

Sur cette hyperbole, le comportement du sol fin saturé est caractérisé par un certain nombre de paramètres mécaniques, raisonnablement indépendants de la position du point représentatif de l'état du sol sur le diagramme, c'est-à-dire de la densité ou de la teneur en eau : paramètres de déformabilité mécanique tels que l'indice de compressibilité œdométrique $C_{c}$; caractéristiques de résistance au cisaillement $c^{\prime}$ et $\phi^{\prime}$; paramètres de modélisation élasto-plastique de type Camclay (compressibilités élastique et plastique $\kappa$ et $\lambda$, paramètre d'état critique $M$, etc.).

Dans l'ouvrage, le défaut de saturation des sols compactés place le point d'état $\left[w, \gamma_{]}\right]$à l'intérieur du domaine borné par l'hyperbole de saturation et ces paramètres sont liés à l'histoire du matériau compacté en place, quí n'est pas forcément identique à celle du compactage en laboratoire. De plus, la densité et la teneur en eau ne suffisent pas pour caractériser les propriétés mécaniques: la structure de l'argile, le mode d'humidification et les déformations de cisaillement exercées lors du compactage engendrent une anisotropie mécanique. Il n'est pas rare d'observer in situ dans les planches d'essais un feuilletage qui réduit fortement la cohésion effective et l'angle de frottement effectif sur le plan horizontal. Ce feuilletage, induit par une humi- 
dification périphérique ou par un cisaillement excessif sous l'action du compacteur ou du trafic de chantier, est à éviter en adoptant des caractéristiques et un mode de compactage adéquat; si un doute subsiste sur son existence, il impose de connaître et de prendre en compte dans le calcul de l'ouvrage également les propriétés de résistance sur le plan horizontal qui contiendrait un tel feuilletage.

A l'intérieur de la zone délimitée par Thyperbole, il n'existe plus de relation entre la densité et la teneur en eau, et un autre paramètre, le degré de saturation $S_{\text {r }}$ est nécessaire pour décrire l'état du sol, selon la relation:

$$
\gamma_{d}=\frac{\gamma_{s}}{1+\frac{w \gamma_{s}}{S_{r} \gamma_{w}}}
$$

Graphiquement, le degré de saturation représente le rapport entre l'abscisse du point considéré et l'abscisse du point ayant le même poids volumique situé sur l'hyperbole de saturation; plus la distance du point considéré à l'hyperbole est grande, plus le sol est désaturé. L'expression 2 montre également que les courbes d'isovaleurs de degré de saturation sont aussi des hyperboles. A proximité de l'hyperbole de saturation, dans une zone limitée par une hyperbole correspondant à un degré de saturation constant élevé, supérieur ou égal à $85 \%$ selon les caractéristiques du sol et en particulier son indice de plasticité, le sol se retrouve dans un état quasi saturé, dans lequel l'air est sous la forme de bulles isolées. Dans cette zone, qui correspond aux branches humides des courbes de compactage, parallèles à l'hyperbole de saturation, la succion initiale - telle que définie plus loin - est quasiment nulle et des surpressions interstitielles dangereuses sont susceptibles d'apparaître lors de la construction d'un remblai homogène. On peut dans ce cas modéliser le comportement du sol comme celui d'un sol saturé par un fluide compressible, en utilisant le concept de contrainte effective élargi au fluide compressible.

Pour des degrés de saturation plus faibles, la phase air devient continue, avec apparition de ménisques eau-air, qui se localisent dans des pores de plus en plus petits au cours d'une désaturation (Fig. 1, voir Delage, 1987). L'équilibre du ménisque impose que la pression du côté concave du ménisque (pression du fluide non mouillant, l'air, soit $u_{\text {I }}$ ) soit supérieure à celle du fluide mouillant situé du côté convexe (pression d'eau $u_{w}$ ). On peut définir une pression capillaire $p_{c}=u_{u}-u_{4}$. qui est positive compte tenu de la remarque faite précédemment sur l'équilibre du ménisque ; en considérant conventionnellement la pression atmosphérique comme nulle, on voit que la pression de l'eau est négative au voisinage du ménisque. En fait, vu les forts niveaux atteints par la différence $u_{n}-u_{u}$ du fait de l'attraction exercée sur les molécules d'eau par les minéraux argileux, mécanisme complémentaire à l'action capillaire décrite précédemment, on utilise fréquemment la notion de succion $s=u_{-}-u_{u}$, qui englobe à la fois les effets capillaires et ceux dus a l'interaction chimique eau-argile (Statement of the review panel, 1965). Cette succion agit comme un lien au contact de deux particules de sol, soit par le biais d'un ménisque pour un sol granulaire, soit en mobilisant également les mécanismes d'adsorption eau-argile, dont l'ampleur est quantifiée macroscopiquement par les limites d'Atterberg; l'effet des paramètres de plasticité sur les courbes de rétention d'eau se révèle ainsi être important (Black, 1962).

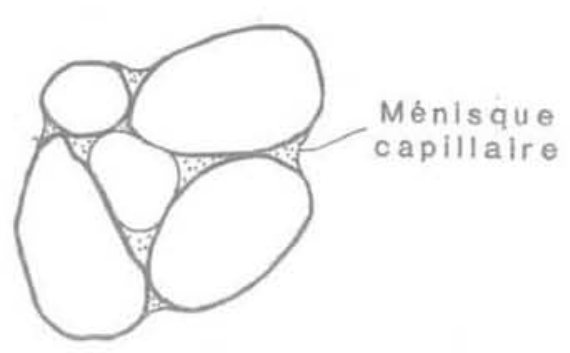

Sol granulaire

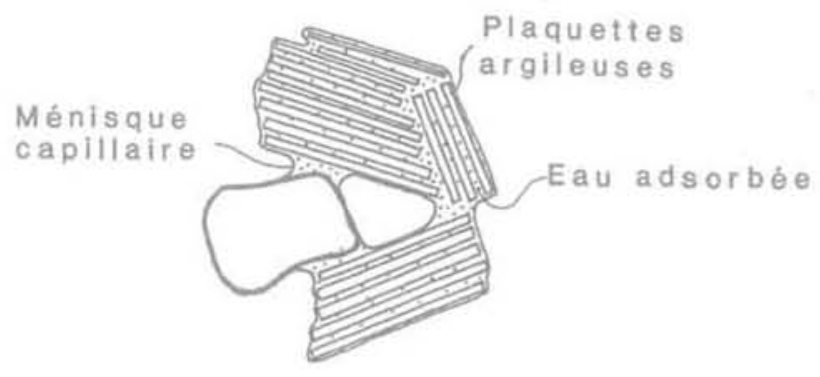

Sol fin

FG.1 Représentation schématique de l'eau au sein d'un sol non saturé (d'après Delage 1987 ) : a) sol granulaire ; b) sol fin.

Scheme of the position of water in an unsaturated soil (after Delage, 1987) : a) sand ; b) fine grained soil.

On peut affirmer en première analyse que, pour un sol donné, la succion est d'autant plus élevée que le degré de saturation est faible, c'est-à-dire que le point caractéristique dans le diagramme de compactage est éloigné de l'hyperbole de saturation. C'est ainsi que le séchage d'un sol, qui se fait à succion croissante. entraînera un déplacement du point caractéristique vers la gauche, et vers le haut si le sol se rétracte; un remouillage, lors duquel la succion décroit, correspondra à un chemin horizontal vers la droite, pouvant descendre dans le cas d'un gonflement; un autre chemin de succion décroissant s'obtient lors d'une compression à teneur en eau constante, qui correspond à un chemin vertical vers le haut, quit rapproche le point de Thyperbole de saturation (Fig. 2). A tous ces points correspondent des caractéristiques mécaniques de déformabilité et de résistance maximales différentes; elles dépendent de la densité et de la teneur en eau, et donc de la succion, qui est une fonction de ces deux paramètres et d'autres caractéristiques du sol, comme l'indice de plasticité.

Le tableau I donne les valeurs de succion mesurées sur divers sols de barrages, compactés à l'optimum Proctor normal. On n'observe pas de corrélation directe entre la succion et un autre paramètre caractéristique ; cependant, on observe que les sols granulaires à faible fraction argileuse $(<10 \%)$ ont des succions de quelques dizaines de $\mathrm{kPa}$; quand la fraction argileuse dépasse $20 \%$, les succions sont supérieures à $100 \mathrm{kPa}$, avec des valeurs maximales voisines de $300 \mathrm{kPa}$.

En fait, la succion des sols compactés peu plastiques ne semble pas très sensible aux variations de densité pour les états significativement désaturés, comme le 


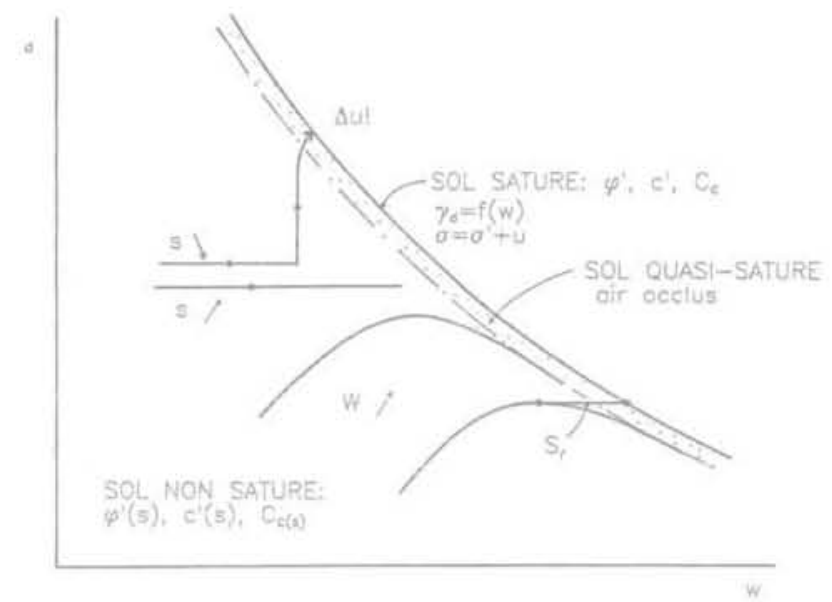

FG. 2 Diagramme de compactage d'un sol. Compaction diagram of a soll.

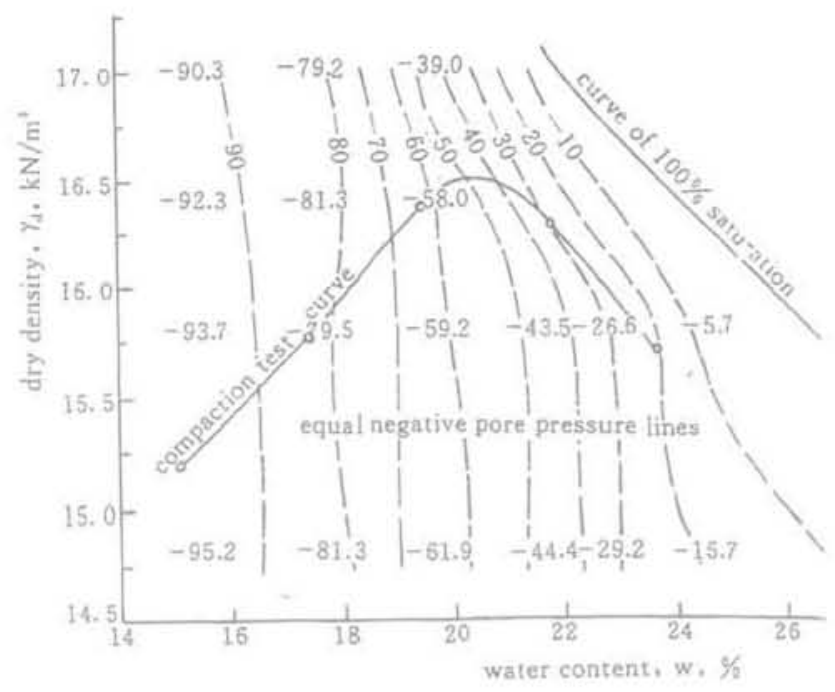

FIG.3 Isovaleurs de succion d'un sol compactè (Li, 1995).

Constant suction curves of a compacted low plasticity loess (Li, 1995)

TABLEAU1 Valeurs de succion de quelques sols compactés à l'optimum (d'après Fry et Delage, 1993).

\begin{tabular}{|c|c|c|c|c|c|c|}
\hline Sol & Auteur & Succion & $w_{\text {ogn }}$ & $\%<2 \mu \mathrm{m}$ & $I_{n}$ & $w_{1}$ \\
\hline Gravier du Chambon & Fleureau & $20 \mathrm{kPa}$ & 10 & 4 & 5 & 26 \\
\hline Lalybont silt & Bishop & $30 \mathrm{kPa}$ & 10 & 4 & & \\
\hline Sable argileux de La Verne & Fleureau & $30 \mathrm{kPa}$ & 18 & 3 & 17 & 36 \\
\hline Gravier du Clou & Fleureau & $50 \mathrm{kPa}$ & 7 & 6 & 2 & 23 \\
\hline Loess & Li & $50 \mathrm{kPa}$ & 20,5 & 35 & 7 & 26 \\
\hline Limon de Jossigny & Delage & $150 \mathrm{kPa}$ & 18 & 35 & 18 & 35 \\
\hline Argile sableuse & USBR & $170 \mathrm{kPa}$ & 17 & 26 & 24 & 40 \\
\hline Mangla clay & Bishop & $270 \mathrm{kPa}$ & 16 & 21 & 17 & 38 \\
\hline Selset clay & Bishop & $350 \mathrm{kPa}$ & 11 & 20 & 17 & 33 \\
\hline Brown clay & Morris & $300 \mathrm{kPa}$ & 29 & 33 & 43 & 68 \\
\hline
\end{tabular}

montre la figure 3 (Li, 1995). Les courbes d'isovaleurs de succion représentées sur le diagramme de compactage de ce loess peu plastique $\left(\mathrm{w}_{1}=26, \mathrm{w}=19,32 \%\right.$ $<2 \mu \mathrm{m})$ sont verticales pour les points suffisamment éloignés de l'hyperbole de saturation. Elles s'inclinent en lui devenant parallèles quand on s'en rapproche. La Figure montre que la succion à l'optimum est proche de $50 \mathrm{kPa}$. Des résultats analogues ont été présentés par Gens et al. (1995) sur le limon de Barcelone $\left(w_{1}=30,5, w_{p}=18,7,24 \%<2 \mu m\right)$.

Les propriétés des sols compactés dépendent également de l'histoire hydromécanique du sol, c'est-à-dire du chemin suivi par le sol dans le diagramme $(\gamma / w)$ pour en arriver à la position considérée. Ces propriétés variables, dont l'évolution est assez mal connue, rendent le problème complexe. C'est probablement la raison pour laquelle on s'est longtemps contenté de considérer les propriétés des échantillons compactés puis saturés. Il est cependant possible à présent de fournir un certain nombre d'éléments complémentaires permettant une meilleure compréhension du comportement mécanique des sols compactés.

\section{3}

\section{Microstructure des sols compactés}

Bien que les techniques développées en pédologie et en géologie de l'ingénieur pour l'étude de la microstructure des sols fins aient fait d'énormes progrès ces vingt dernières années, il ne semble pas que leur exploitation en géotechnique pour une meilleure compréhension du comportement hydromécanique des sols ait été suffisamment développée. Ceci vaut en particulier pour les sols compactés, qui ont pourtant fait l'objet d'un des premiers modèles de microstructure de sol dans un contexte géotechnique, avec la théorie de Lambe (1958a). Ce modèle est encore régulièrement évoqué dans les ouvrages d'enseignement, il est à la base des notions de structures floculée et dispersée (Fig. 4). Ce modèle repose sur la théorie de la double couche (voir Mitchell, 1993), selon le principe suivant : un sol du côté humide de l'optimum possède suffisamment d'eau pour que la double couche d'ions attirés électriquement par les plaquettes argileuses puisse se développer intégralement. 
Dans de telles conditions, l'action prédominante entre deux plaquettes voisines serait une action de répulsion due à cette double couche, et il en résulterait après compactage un arrangement de particules avec des orientations essentiellement parallèles, définissant une structure dispersée. Du côté sec, la quantité d'eau est insuffisante pour un développement complet de la double couche. La distance entre deux feuillets argileux est plus faible, ce qui confère aux actions d'attraction de Van der Waals un rôle prédominant, et conduit ả la floculation, avec davantage de liaisons bord-face, et agrégation des plaquettes les unes avec les autres; il en résulte la formation de flocons qui, comprimés les uns contre les autres constituent la structure globale. Ce modèle est basé sur une théorie initialement développée sur des suspensions colloïdales d'argiles dans l'eau (Van Olphen, 1977), et il n'évoque que faiblement les phénomènes capillaires et leur influence mécanique d'attraction entre les particules.

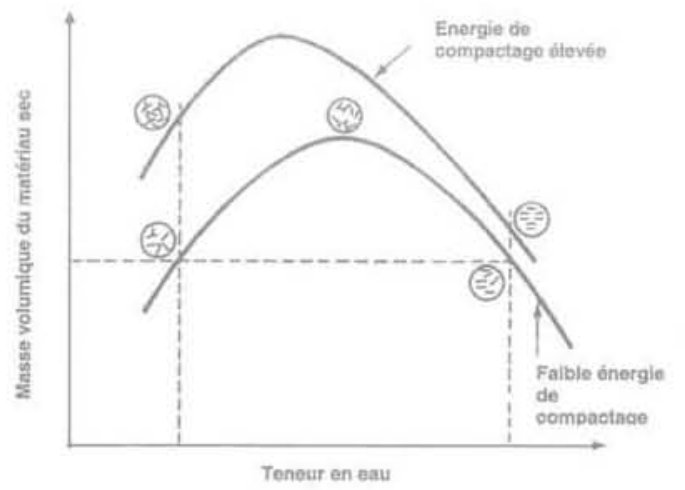

FG.4 Modièle de Lambe (1958a) pour la structure des sols compactés.

Lambe's model (1958a) of the structure of compacted soils.

L'observation en microscopie électronique à balayage sur des échantillons déshydratés avec soin pour éviter les effets dus à la rétraction lors du séchage permet d'identifier des différences essentielles entre deux échantilions compactés à la mème densité, mais situés de part et d'autre de l'optimum, du côté humide et du côté sec (Delage et al. 1996). La figure 5 montre les résultats obtenus sur un limon des plateaux de l'Est parisien, le limon de Jossigny, dont les caractéristiques de compactage sont illustrées par la figure 6, et précisées dans le tableau II. Bien que la densité des échantillons testés soit identique $\left(\gamma_{d}=16 \mathrm{kN} / \mathrm{m}^{3}\right)$, les deux échantillons présentent un aspect singulièrement différent: l'échantillon compacté du côtẻ sec présente un aspect granulaire marqué, avec des agrégats de particules limoneuses de diamètre compris entre 10 et 20 microns. Les plaquettes argileuses, qui correspondent à une proportion non négligeable de $38 \%$ en masse, ne sont que peu apparentes sur le cliché. En fait, elles sont fortement collées sur les grains, et leur confèrent un aspect de surface déchiqueté. L'apparence du sol compacté du côté humide est foncièrement différente. Les grains de limon ne sont plus apparents, on les devine derrière un voilage constitué des plaquettes argileuses hydratées (on a ici $w=21,5 \%$ ). La fraction argileuse massique de $38 \%$ est extrêmement présente en termes de volume, puisqu'elle est en quantité suffisante pour constituer une matrice qui enrobe complètement les grains de limon. L'échantillon compacté au Proctor normal a une apparence plutồt granulaire, à l'instar de l'échantillon sec, mais avec une évidence moindre de l'organisation en agrégats.

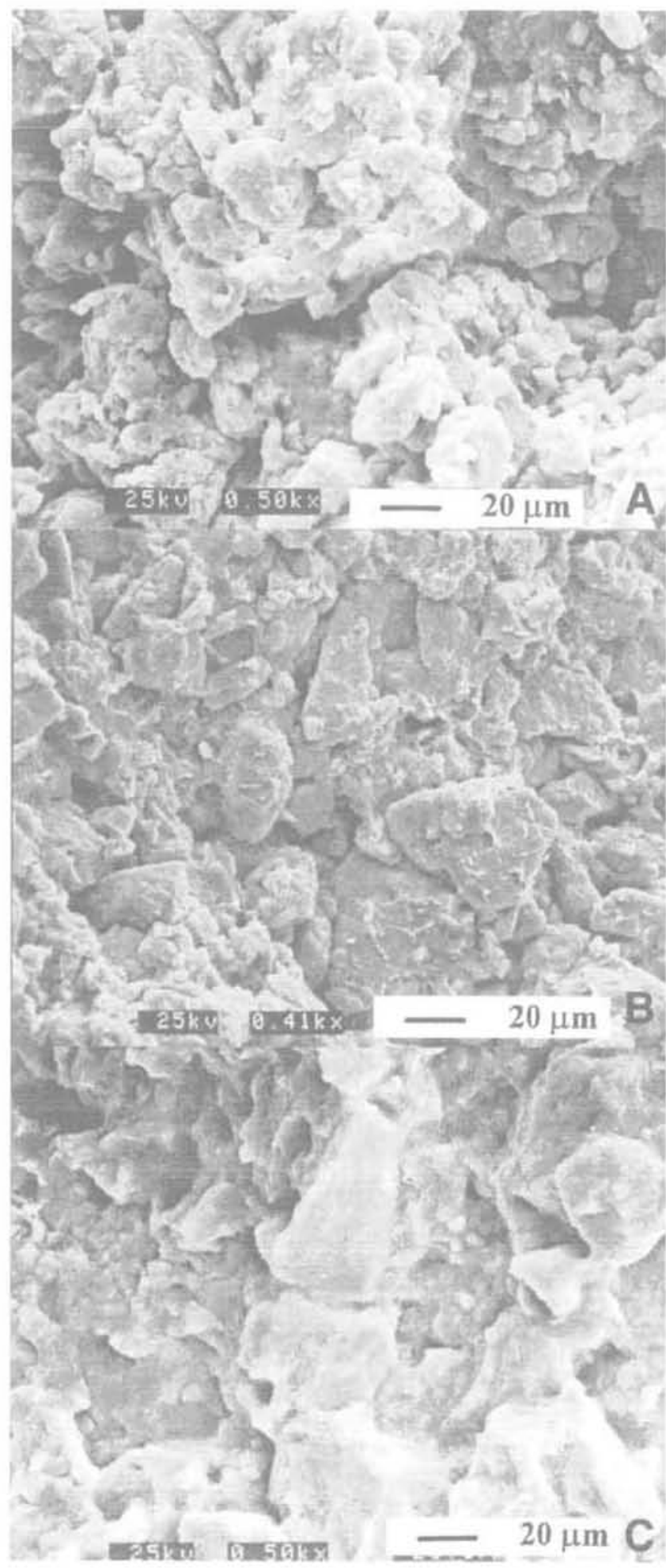

FG.5 Microstructure du limon de Jossigny au microscope électronique à balayage (Delage et al., 1996) : a) côté sec; b) optimum Proctor normal ; c) côté humide. Microstructure of the Jossigny silt, observed using the scanning electron microscope (Delage et al., 1996) : a) dry side ; b) wet side ; c) Proctor normal optimum. 
tABLEAUII Caractéristiques du limon de Jossigny.

\begin{tabular}{|l|c|c|c|c|c|c|c}
\hline$W_{i}$ & $W_{\mathrm{p}}$ & $\mathrm{I}_{\mathrm{p}}$ & $<2 \mu \mathrm{m}$ & $>60 \mu \mathrm{m}$ & $\begin{array}{c}\text { Teneur en eat } \\
\text { aे Loptimum }\end{array}$ & $\begin{array}{c}\text { Poids volumique } \\
\text { a loptimum }\end{array}$ & $\begin{array}{c}\text { Poids volumique } \\
\text { des solides }\end{array}$ \\
\hline $37 \%$ & $19 \%$ & $18 \%$ & $34 \%$ & $10 \%$ & $18 \%$ & $16,7 \mathrm{kN} / \mathrm{m}^{3}$ & $27,2 \mathrm{kN} / \mathrm{m}^{3}$ \\
\hline
\end{tabular}

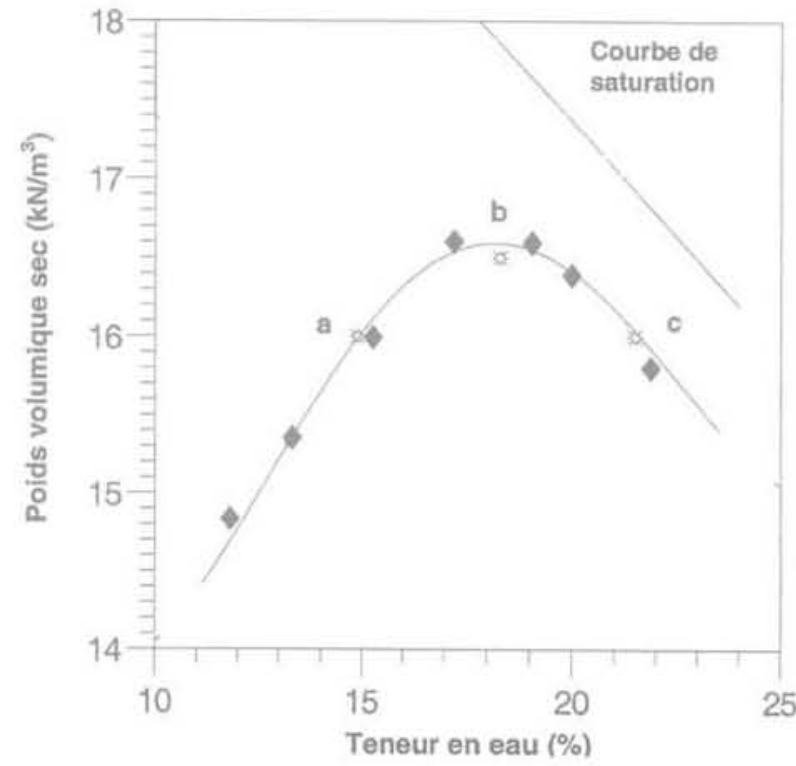

F16.6 Courbe de compactage du limon de Jossigny.

Compaction curve of the Jossigny silt.

Ces observations sont confirmées par les mesures de porosimétrie au mercure présentées en figure 7. La porosimétrie au mercure (Pellerin, 1979) permet de définir approximativement la distribution de la taille des pores d'un milieu donné ; on peut affirmer, pour simplifier, qu'elle est aux pores ce que la granulométrie est aux grains du sol. On observe pour le sol compacté du côté sec une double distribution définissant une famille de gros pores qui correspondent à la porosité inter-agrégats observée sur la figure 5 a, et une famille de pores plus petits correspondant aux pores intra-agrégats. Le rayon lu ici des pores inter-agrégats $(4 \mu \mathrm{m})$ est plus faible que celui observée en microscopie $(20 \mu \mathrm{m})$. Ceci est caractéristique de la porosimétrie au mercure, qui ne sait déterminer que des rayons d'accès aux pores, qui peuvent ètre largement inférieurs aux dimensions réelles du pore lui-même. La courbe de l'échantillon compacté du côté humide est en revanche unimodale. Ceci correspond à la prédominance des particules argileuses observée sur la figure $5 c$. En d'autres termes, le mercure n'a reconnu lors de son intrusion que les pores de la matrice argileuse au sein de laquelle sont baignés les grains de limon. D'après la porosimétrie, l'accès à ces pores à un rayon de $0,45 \mu \mathrm{m}$. L'échantillon compacté à l'optimum a une courbe intermédiaire entre les deux précédentes. Une seule famille est apparente, comparable à la porosité intra-agrégats de l'échantillon sec, mais une partie non négligeable de la porosité totale ( $40 \%$ ) est mal classée, avec des diamètres variant entre 0,5 et $50 \mu \mathrm{m}$. Cette caractéristique peut être associée à un certain désordre, qu'on observe sur la photo de la figure $5 \mathrm{~b}$.
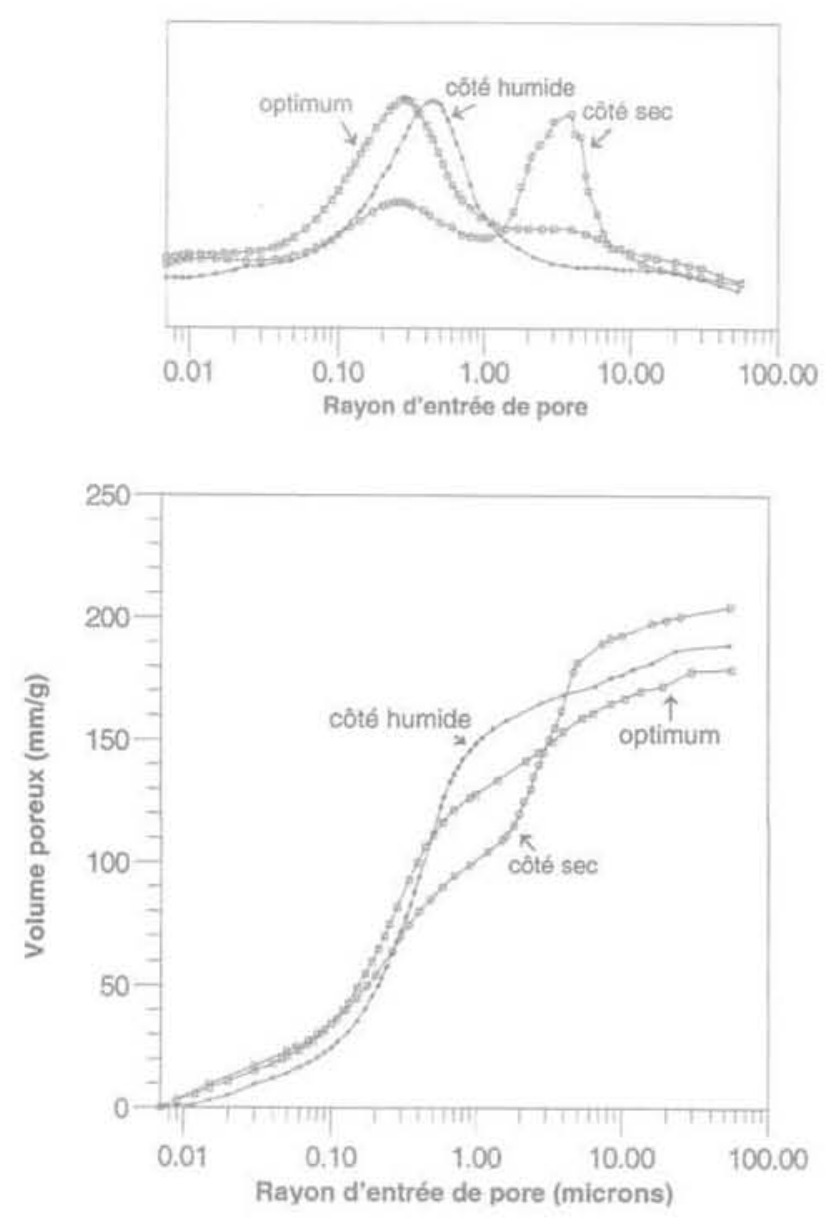

FG.7 Courbes porosimétriques du limon de Jossigny compacté (Delage et al. 1996).

Pore size distribution curves of the compacted Jossigny silt (Delage et al. 1996).

Ces résultats, qui montrent que le modèle théorique de Lambe (1958-a) mérite d'être reconsidéré à la lumière d'observations directes, permettent un certain nombre de considérations d'ordres mécanique et hydraulique. Ils confirment les observations de Lambe (1958-b) montrant, à l'aide d'essais de perméabilité sur échantillons compactés puis inondés, que la perméabilité du sol compacté du côté sec est supérieure à celle du sol côté humide, du fait de la présence de pores plus grands entre les agrégats, avec une diminution de deux ordres de grandeur du coefficient de perméabilité en saturé d'une argile sableuse $\left(10^{-7}\right.$ à $\left.10^{-9} \mathrm{~m} / \mathrm{s}\right)$ entre un point situé du côté sec $\left(\mathrm{w}=12 \%, p_{\mathrm{d}}=1,85 \mathrm{Mg} / \mathrm{m}^{3}\right.$, $\left.\mathrm{S}_{\mathrm{f}}=63 \%\right)$ et du côté humide $\left(15 \%, 1,95 \mathrm{Mg} / \mathrm{m}^{3}\right.$, $\mathrm{S}_{\mathrm{f}}^{r}=95 \%$ ) de l'optimum Proctor $\left(14 \%, 1,98 \mathrm{Mg} / \mathrm{m}^{3}\right.$, $\mathrm{S}_{\mathrm{S}}=92 \%$ ). La valeur de $10^{-9} \mathrm{~m} / \mathrm{s}$ est atteinte à partir d'une teneur en eau $\mathrm{w}_{\text {opt }}+1(\mathrm{w}=15 \%)$, la perméabilité 
restant approximativement constante à cette valeur pour tous les points de la courbe Proctor qui longe I'hyperbole. Ces tendances ont été confirmées par Garcia-Bengochea et al. (1978), Juang et Holtz (1985), qui ont intégré des écoulements de type Poiseuille dans différents modèles de milieux poreux basés sur les courbes porosimétriques. L'effet de la porosité interagrégats se fait clairement sentir. Si l'on considère que les pores emplis d'eau sont les pores de plus petites dimensions, on voit que du côté sec de l'optimum, avec un degré de saturation de $60 \%$, seuls les agrégats sont hydratés, les pores intergranulaires étant emplis d'air. Les observations microstructurales précédentes montrent également que l'échantillon humide doit avoir un comportement proche de celui d'un sol fin, du fait de la prédominance volumique de la phase argileuse hydratée. Dans une telle microstructure, la quantité d'air, qui correspond ici à un degré de saturation de $86 \%$, se trouve sous forme de bulles occluses, qui sont difficiles à expulser par compression, du fait de l'absence de connexions entre elles. C'est ainsi qu'il est impossible, à teneur en eau constante, d'atteindre par compactage l'état saturé. Le parallélisme entre la branche humide de la courbe Proctor et l'hyperbole de saturation est à relier à ce degré de saturation résiduel en air.

C'est dans ce type de microstructure que s'initie la génération des surpressions interstitielles, par compression des bulles d'air occluses, jusqu'à atteindre une valeur positive de la pression d'eau. Une autre conséquence de cette situation observée lors de la réalisation des ouvrages est le matelassage : le passage de l'engin de compactage comprime élastiquement les bulles contenues dans le sol, qui reprennent leur volume initial après le passage de l'engin.

D’un point de vue mécanique, le sol compacté sec aura un comportement de type granulaire frottant, alors que le sol compacté humide comporte une matrice argileuse, qui jouera un rôle de lubrifiant, avec un frottement macroscopique dit " intergranulaire » plus faible. Du fait de l'absence de la classe de pores intergranulaire et de la plus grande densité qui en résulte, l'échantillon compacté à l'optimum Proctor aura, une fois saturé, les meilleures caractéristiques mécaniques, tant en termes de déformabilité que de résistance maximale.

A l'échelle microscopique, ces observations confirment le mécanisme observé sur chantier lors de la mise en œuvre de massifs de sol compacté : un sol plus sec sera constitué de mottes plus rigides et plus résistantes, dont il restera une trace après le passage de l'engin de compactage. Les vides d'air entre les mottes pourront subsister, mais pourront engendrer des tassements différés lors de la première mise en eau d'un barrage ou lors de redistributions d'eau dues à la capillarité et éventuellement alimentées par la nappe. Inversement, un sol compacté humide paraît plus malléable et formera après le passage de l'engin un massif plus continu et déformable ; dans les massifs compactés destinés à assurer des fonctions d'imperméabilité (barrages en terre, tapis imperméables pour le confinement de déchets), on favorise le pétrissage (Kouassi et al., 2000), qui augmente le degré de destruction des agrégats, par l'utilisation de compacteur à pieds dameurs ou à pieds de mouton, ce qui permet de réduire encore plus la perméabilité.

L'optimum Proctor se retrouve ainsi être la teneur en eau pour laquelle les mottes ne sont pas trop mal- léables, mais également pas trop rigides pour pouvoir être cassées en favorisant l'expulsion de l'air lors du passage de l'engin, en conférant au matériau une densité macroscopique maximale. La figure 8 (Cabot et Le Bihan, 1993) décrit ce phénomène au niveau macroscopique, en montrant l'influence de la teneur en eau et de l'énergie de compactage sur la constitution du matériau compacté. Ces notions de microstructure des sols compactés ont été utilisées dans la pratique pour la réalisation d'écrans étanches ou de revêtements imperméables pour les lacs artificiels ou les dépôts de déchets liquides ou solides (Auvinet et Espinoza, 1979; Benson et Daniel, 1990),

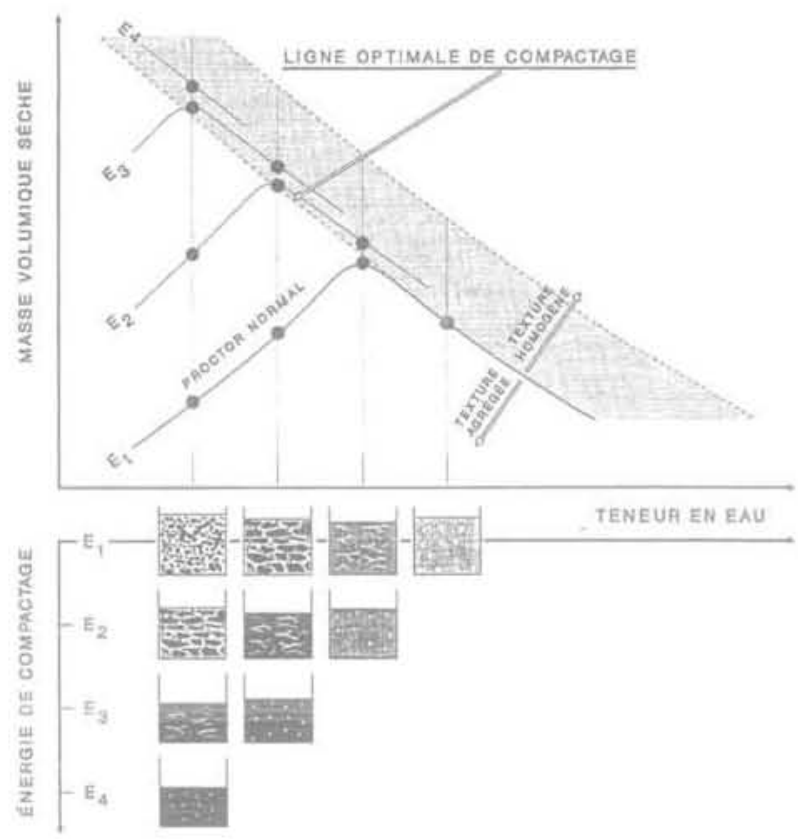

FG. 8 Evolution de la structure d'une argile compactée en fonction de la teneur en eau et de l'énergie de compactage (Cabot et Le Bihan, 1993).

Changes in the structure of a compacted clay, as a function of the water content and density (Cabot et Le Bihan 1993). 


\section{Microstructure et compression volumique}

L'évolution de la microstructure d'un sol compacté du côté sec peut être observée en utilisant les résultats de Sridharan et al. (1971), qui ont réalisé des essais porosimétriques sur des échantillons compactés à la même teneur en eau, mais à des indices des vides décroissants obtenus en appliquant des contraintes de compactage de plus en plus fortes. On observe sur la figure 9 que la diminution de volume se fait par l'écrasement des plus gros pores emplis d'air, une contrainte de plus en plus forte affectant des pores de plus en plus petits. Ce phénomène a déjà été observé sur des argiles molles saturées (Delage et Lefebvre 1984). Il explique également le fait que le tassement des sols non saturés, où l'air est continu, se fait quasi instantanément par expulsion et compressibilité immédiate de l'air, et réorganisation locale des ménisques d'eau, sans aucun transfert liquide.

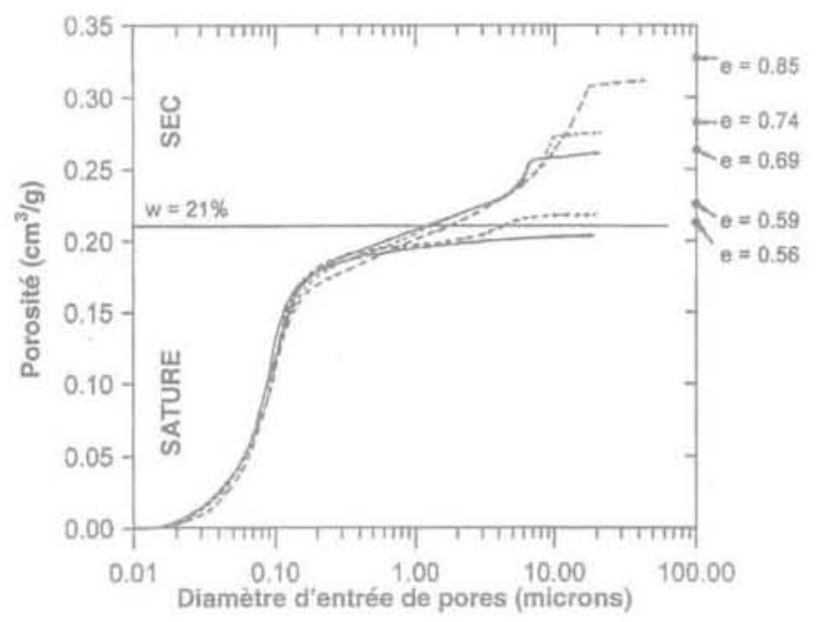

F16.9 Effet de la compression sur la porosimétrie d'une kaolinite compactée (Sridharan et al. 1971 .

Effect of compression on the pore size distribution curve of a compacted kaolinite (Sriciharan el al. 1971).

Ce phénomène est illustré par les courbes dé tassement en fonction du temps obtenues en réalisant un essai de compression œdométrique à teneur en eau constante sur un limon de Jossigny compacté. Ce type d'essai est assez simple à réaliser dans un laboratoire de mécanique des sols classique, pourvu que les précautions nécessaires pour lutter contre l'évaporation de l'eau soit prises, et qu'on utilise des pierres poreuses sèches afin de ne pas modifier la teneur en eau du sol. Il présente l'intérêt de donner la compressibilité réelle du sol compacté, qui est très différente de celle qui serait obtenue après saturation. Le dispositif idéal pour ce type d'essai a été présenté par Yoshimi et Osterberg (1963). Une variante adaptable à des cedomètres classiques est illustrée en figure 10. Il consiste à isoler l'éprouvette de l'air ambiant par un film plastique reliant le piston à la cellule, et à saturer de vapeur d'eau l'ambiance à l'intérieur de la cellule cedométrique en y plaçant un petit récipient empli d'eau. Un contrôle effectué avant et après un essai d'une semaine montre une diminution de la teneur en eau du sol inférieure à 0,5 point. Après la phase initiale de tassement instantané, les courbes de la figure 11 ont une allure linéaire en fonction du logarithme du temps, ce qui montre que le fluage des sols non saturés à teneur en eau constante obéit aux mémes règles que la plupart des autres matériaux. Ces courbes montrent également qu'un essai de compressibilité à teneur en eau constante peut être réalisé assez rapidement, puisqu'il n'est pas nécessaire d'attendre, comme dans le cas saturé, la dissipation de surpressions interstitielles. Il semble qu'une durée de l'ordre de 30 minutes, analogue à celle adoptée par Muravama et al. (1984) pour l'étude du fluage des sables, soit suffisante pour un incrément de chargement.

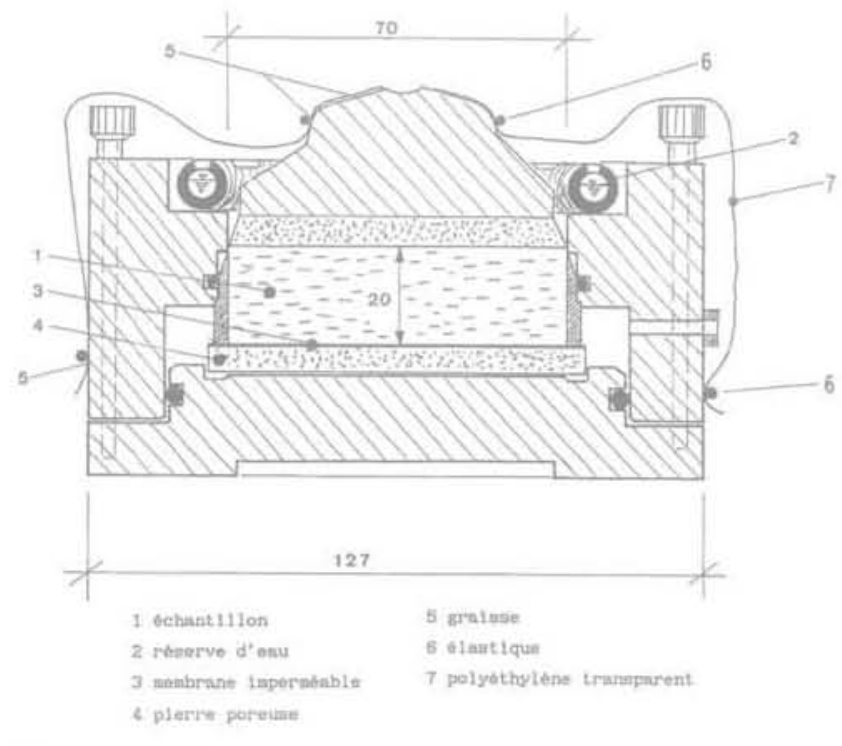

FG. 10 Cellule œdométrique pour essai à teneur en eau constante : 1) échantillon ; 2) réserve d'eau ; 3) membrane imperméable : 4) pierre poreuse ; 5) graisse ; 6) élastique ; 7) film imperméable transparent.

Oedometer cell used for constant water content tests : 1) sample; 2) water reservoir ; 3) impermeable membrane : 4) porous stone ; 5) grease ; 6) o-ring: 77 impermeable polythene film.

Dans leur étude de la compressibilité des sols compactẻs à teneur en eau constante, Yoshimi et Osterberg (1963) ont mis en évidence un certain nombre de traits de comportement caractéristiques. En montrant que la cinétique du tassement était indépendante de l'épaisseur de l'échantillon œdométrique, ils ont confirmé l'absence de transfert d'eau au sein de l'éprouvette non saturée en cours de compression. En testant à l'œdomètre un échantillon préalablement compacté statiquement à une contrainte connue, ils ont montré que l'échantillon gardait précisément la mémoire de sa contrainte de compactage. Ceci est illustré sur la figure 12 dans le cas d'une éprouvette de limon de Jossigny compacté à une teneur en eau de 22 $\%$ et une densité de $15,6 \mathrm{kN} / \mathrm{m}^{3}$ en appliquant lors du compactage statique une contrainte verticale maximale de $220 \mathrm{kPa}$. La forme de la courbe montre bien un comportement surconsolidé pseudo-élastique en dessous de cette valeur, et normalement consolidé plastique au-dessus. La nature surconsolidée des sols compactés a été confirmée de manière plus approfon- 
$\mathrm{t}$ (minutes)

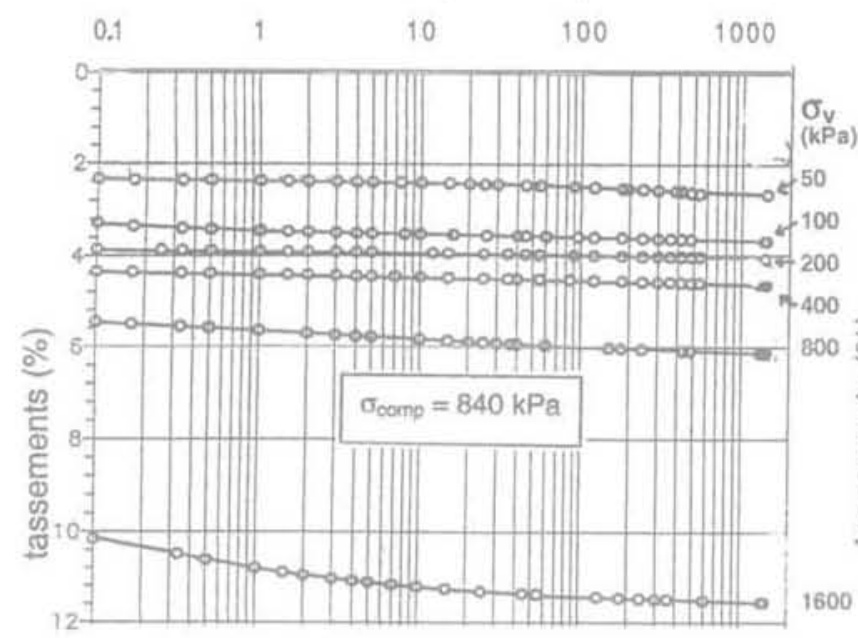

$\mathrm{t}$ (minutes)

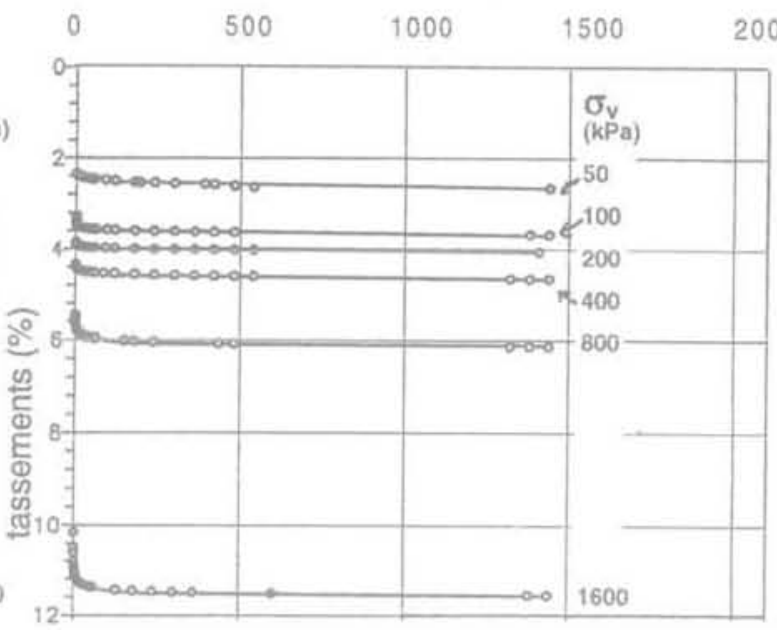

FG.11 Courbes de tassement en fonction du temps lors d'un essai à teneur en eau constante sur sol compacté (limon de Jossigny) : a) logarithmique ; b) linéaire ( $\left.w=15,3 \%, \gamma_{t}=16,1 \mathrm{kN} / \mathrm{m}^{3}\right)$.

Settlement as a function of time, observed in a constant water content compression test on a compacted sample (Jossigny silt) ; a) logaritmic; b) linear $\left(w=15.5 \%, \gamma_{\mathrm{c}}=16.1 \mathrm{kN} / \mathrm{m}^{2}\right)$ :

die dans un cadre élasto-plastique (Alonso et al., 1990) en tenant compte également des aspects déviatoriques du comportement dans Cui et Delage (1996). Au sein d'un remblai, il existe donc au niveau des couches supérieures une épaisseur de sol compacté ou la contrainte verticale est inférieure à la contrainte de compactage, qui ne présentera que des tassements faibles correspondant à la zone surconsolidée définie sur la figure 12. Pour les couches situées à une plus grande profondeur à partir de la surface du talus du remblai, le sol est comprimé à des niveaux de contrainte supérieure à celle qu'il a vécu lors du compactage, et il subira des tassements plus forts lors de la

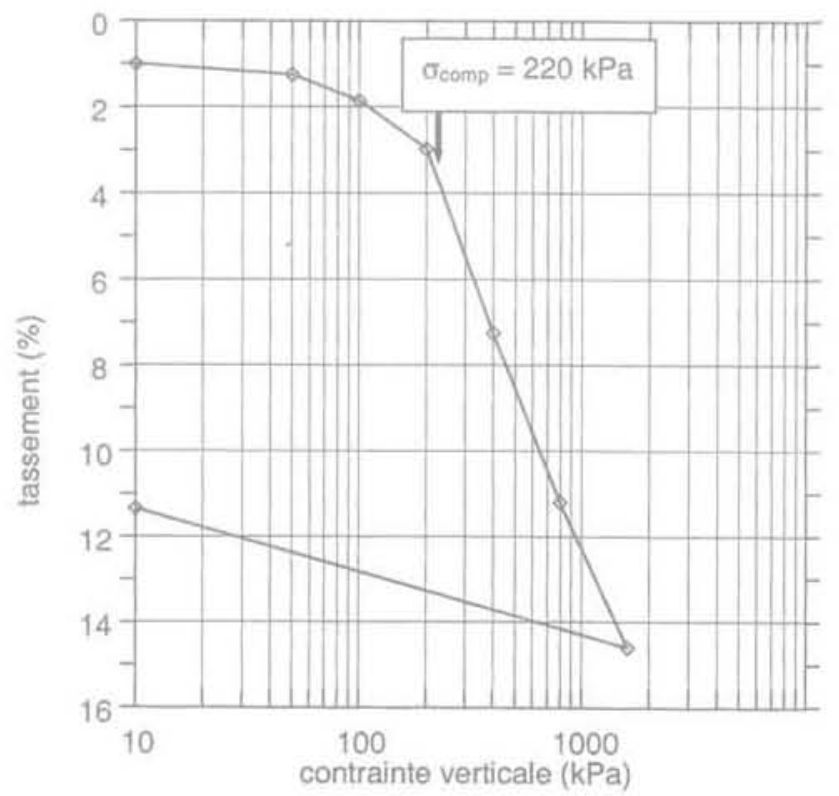

HG. 12 Compression cdométrique d'un sol compacté flimon de Jossigny, $w=22 \%, \gamma$ $\left.=15,6 \mathrm{kN} / \mathrm{m}^{3}\right)$ : mémoire de la contrainte de compactage $\left(\sigma_{\text {cnmp }}=220 \mathrm{kPa}\right)$.

Oedometer compression of a compacted soil (Jossigny silt, $\left.w=22 \%, \gamma_{d i}=15.6 \mathrm{kN} / \mathrm{m}^{3}\right)$ : memory of the compaction stress $\left(\sigma_{\text {ctimp }}=\right.$ $220 \mathrm{kPa}$ ). mise en place des couches supérieures. Il existe ainsi une épaisseur critique, de l'ordre de 11,5 m dans le cas du sol de la figure 12, au-delà de laquelle des déformations de tassement d'un ordre supérieur sont à attendre. Cette épaisseur a son importance en termes de générations de pressions interstitielles positives en cours de construction, en particulier pour les ouvrages en terre (barrages, remblais) homogènes de hauteur comprise entre 10 et $30 \mathrm{~m}$.

La figure 13 montre l'effet conjugué de la teneur en eau et de la densité sur la compressibilité d'un sol compacté. On observe en comparant les courbes 1 et 2 (poids volumiques secs voisins - 16,1 et $15,95 \mathrm{kN} / \mathrm{m}^{3}$ et teneurs en eau de 16 et $18 \%$ respectivement) qu'à

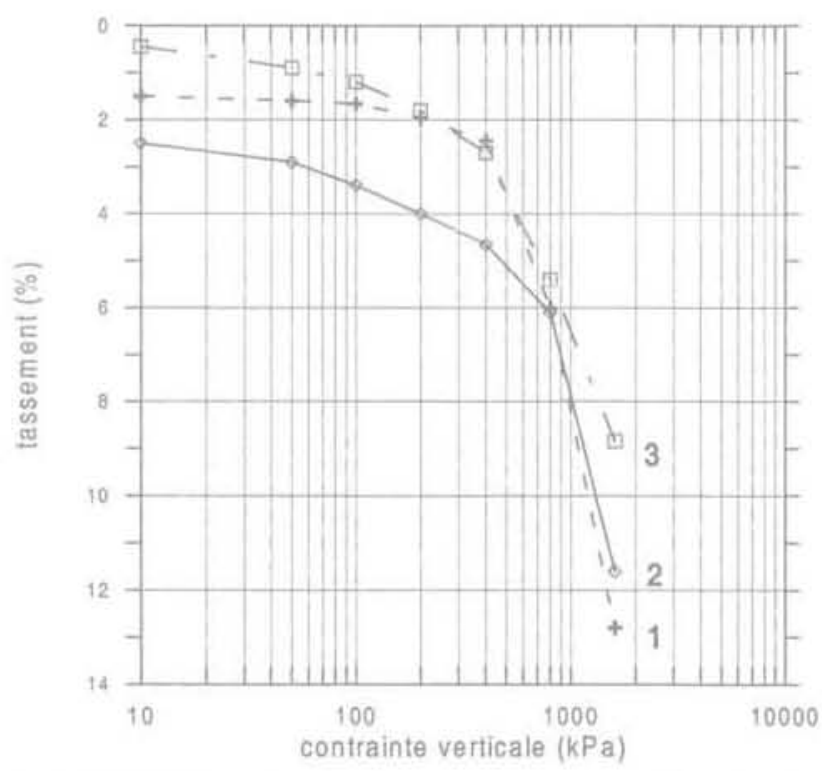

Fig. 13 Effets de la teneur en eau et de la densité sur la compressibilité d'un sol compacté (limon de Jossigny).

Effects of water content and density on the compressibility of a compacted (soll Jossigny silt). $1 \gamma_{w}=16.10 \mathrm{kN} / \mathrm{m}^{3}, w=16 \%, 2 \gamma_{w}=15.95 \mathrm{kN} / \mathrm{m}^{3}$, $w=18 \%, 3 \gamma_{w}=16.55 \mathrm{kN} / \mathrm{m}^{3}, w=20 \%$. 
densité égale, le sol le plus humide est le plus compressible. La courbe 3 montre cependant que l'influence de la densité est prépondérante, puisque l'éprouvette compactée à un poids volumique sec de $16,55 \mathrm{kN} / \mathrm{m}^{3}$ et à une teneur en eau de $20 \%$ est moins compressible que les deux précédentes. On note également sur la figure que le tassement instantané est d'autant plus faible que la teneur en eau est élevée.

\section{5}

\section{Comportement volumique des sols compactés}

A côté de l'essai à teneur en eau constante décrit précédemment, deux autres types d'essais sont réalisables sur une éprouvette de sol compacté (Fig. 14). Le plus simple consiste à remouiller l'éprouvette sous une faible contrainte, pour ensuite procéder à un essai de compression classique de l'éprouvette inondée. En fait, la condition de saturation totale n'est pas nécessairement remplie lors de l'inondation, qui ramène en revanche la valeur de la succion exactement à zéro. Des piégeages de bulles d'air peuvent faire en sorte que le degré de saturation soit légèrement inférieur à 100\%, et cela se traduit par un tassement instantané lors des phases de chargement œedométrique, qui correspond à la compression des bulles d'air occluses. Par rapport à un chargement à teneur en eau constante, idéalement représenté en figure 14 (ab), le remouillage sous faible contrainte (ac) se traduit, dans le cas d'un sol non gonflant considéré ici, par une légère augmentation de volume correspondant au relâchement de la succion depuis sa valeur initiale $s_{0}$ jusqu'à zéro. L'expérience montre que la phase de compression qui suit (cd) engendrera toujours des déformations supérieures à celles observées à teneur en eau constante. De nombreux travaux expérimentaux ont montré que, sous une contrainte donnée suffisamment élevée et pour un sol non gonflant, c'est à l'état saturé qu'est obtenu le plus faible indice des vides d'un sol. Cette constatation est à la base de la méthode dite du double œdomètre développée par Jennings et Knight (1957) pour la détermination du potentiel d'effondrement des sols.

Un troisième essai possible est l'essai à succion contròlée (ae), égale à la succion initiale de l'échantillon compacté. Dans ce cas, les déformations observées sont plus faibles qu'à teneur en eau constante (Fig. 14a et b). A teneur en eau constante, l'échantillon comprimé se rapproche de la saturation et voit sa succion décroître, ce qui correspond à un ramollissement. La valeur constante de la succion imposée par son contrôle empêche un tel ramollissement, ce quí explique une déformabilité moindre. La réalisation de ce type d'essai est plus délicate. Le plus souvent, on utilise la technique dite de translation d'axe, dans laquelle un dispositif particulier dû à Richards (1941) permet d'appliquer au sein de l'échantillon une contre-pression d'air $u_{0}$ positive tout en maintenant la pression d'eau $u_{w}$ égale a la pression atmosphérique, prise conventionnellement pour nulle. On obtient bien ainsi une condition de succion $\sigma=u_{s}-u_{w}$ positive et égale à la valeur de la pression d'air imposée. Barden et al. (1969) présentent de tels essais œedométriques à succion contrôlée. Une autre technique consiste à mettre le sol au contact d'une membrane semi-perméable derrière laquelle circule une solution aqueuse de molécules de polyéthylène glycol (PEG) de grandes dimensions (Kassiff et Ben Shalom, 1971; Delage et al., 1987). Comme la membrane est perméable à l'eau mais ne laisse pas passer les molécules, elle permet l'application d'une succion osmotique d'autant plus élevée que la concentration en PEG est forte. On a récemment pu étendre la gamme d'application de cette technique à des succions de l'ordre de $10 \mathrm{MPa}$ (Delage et al., 1998). L'intérêt des essais à succion contrôlée est fondamental, dans la mesure où il permet l'investigation séparée des effets respectifs des deux variables de contraintes utilisées en mécanique des sols non saturés, qui sont la contrainte moyenne totale nette $p-u_{\mathrm{i}}$, et la succion $\mathrm{u}_{-}-\mathrm{u}_{\mathrm{w}}$ (Coleman 1962, Fredlund et Morgenstern 1977). Une dernière remarque concernant les essais cedométriques à succion contrôlée concerne le mode de chargement. L'utilisation classique d'cedomètres avec chargement rapide et doublement de la charge à chaque incrément s'est en fait révélée inadaptée pour les sols non saturés. Pour les sols saturés, ce type de chargement correspond à un chargement progressif du squelette, qui intervient lors de la dissipation des pressions interstitielles. Pour un sol non saturé, il s'agit au contraire d'un mode brutal, qui se traduit par un tassement instantané, une expulsion d'air et une diminution soudaine de la succion, qui est ensuite ramenée à sa valeur initiale par le système de contrôle. L'expérience a montré que cette régulation pouvait nécessiter une dizaine d'heures (Delage et De Silva, 1993). Il convient donc d'adopter un mode de chargement lent de type essai drainé, afin qu'en tout instant la variation de succion engendrée par la compression soit régulée par le système, et ainsi maintenue à la valeur souhaitée (Cui et Delage, 1996). Il est donc préférable de réaliser les essais de compression œdométrique à succion contrôlée à vitesse constante lente ( $2 \mu \mathrm{m}$ par minute) sur une presse triaxiale. Un tel essai ne dure en fait qu'une journée, ce qui est intéressant par rapport à la procédure classique.

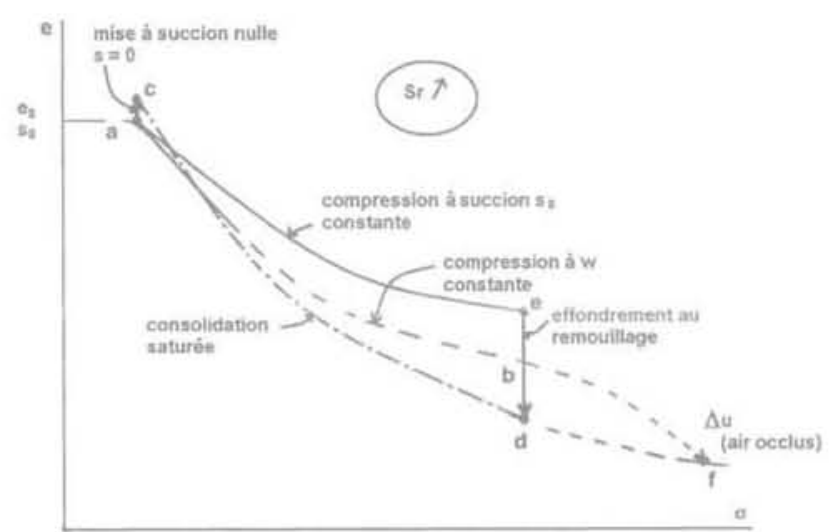

FiG. 14 Divers types d'essais de compression réalisables sur un sol compacté (à degrẻ de saturation croissant).

Various possible compression tests on a compacted soil (degree of saturation increasing).

Comme on l'a vu, les différentes réponses possibles d'un sol compacté sont représentées en figure 14. Les différents indices des vides obtenus sous la contrainte $\sigma$ (points e, b, d) selon le chemin suivi (ae : succion constante, ab teneur en eau constante et acd : inondation et compression à succion nulle) permettent d'introduire le phénomène d'effondrement, qui se produit quand la succion est réduite à zéro par inondation sous une charge $\sigma$ constante, à partir des points e ou b. Cette 
diminution de succion des échantillons en e ou b engendre un tassement qui aboutit au point de da courbe de compression à succion nulle.

Par ailleurs, on observe quaux fortes contraintes, les deux courbes à teneur et succion constante finissent par rejoindre la courbe saturée. En d'autres termes, la contrainte est toujours capable d'atteindre un niveau à partir duquel son action est prépondérante par rapport à celui de la succion, que celle-ci soit constante ou non. Cette intersection correspond, dans le cas de la construction des barrages, au début de la génération des pressions interstitielles positives.

Il est possible de représenter les courbes de la figure 14 dans un diagramme indice des vides - contrainte nette en utilisant la succion comme troisième variable. On obtient alors la représentation graphique en trois dimensions de la figure 15, qui n'est autre que la surface d'état définie par Matyas et Radhakrishna (1968). Ces auteurs ont réalisé de nombreux essais suivants différents chemins de contrainte et de succion sur un sol compacté, et ils ont montré que, dans des conditions de degré de saturation croissante, les points obtenus expérimentalement se situaient tous sur cette surface. Les essais correspondant étaient réalisés soit par chargement à succion constante, soit par remouillage sous contrainte constante. Comme on l'a vu, les essais à teneur en eau constante sont également situés sur cette surface. Cette notion de surface d'état constitue en fait la première modélisation publiée capable de décrire, en conditions de saturation croissante, les variations volumiques d'un sol compacté en fonction des changements de contrainte et de succion. La première implantation de ce concept dans un code de calcul aux éléments finis pour la modélisation des barrages en terre est due à Alonso et al. (1988). Cette notion est également utilisée dans le code U-Dam (Nanda et al., 1993), qui sera décrit dans un article suivant. On observe qu'il existe une surface d'état unique pour une densité et une teneur en eau initiales données, et on a vu que les variations de densité ont une influence importante sur les propriétés de compressibilité des sols non compactés. Idéalement, une grande dispersion des densités au sein d'un ouvrage devrait inciter à étudier leur effet sur la forme de la surface d'état.

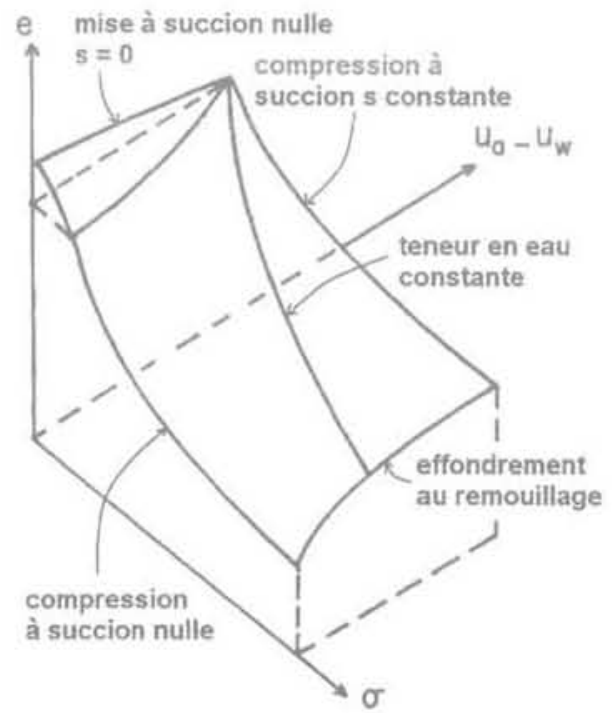

AG. 15. Surface d'état d'un sol compacté (d'après Matyas et Radhakrishna, 1968).

State surface of a compacted soil (after Matyas et Radhakrishna, 1968).
6

\section{Résistance au cisaillement}

Les résultats de la figure 16 , obtenus avec un appareil triaxial à succion contrôlée par un dispositif osmotique (Delage et al,, 1987), illustrent les aspects déviatoriques du ramollissement dû à la diminution de la succion, autant en termes de module de cisaillement que de résistance maximale. Ils montrent comment, sous un état de contrainte totale constant, une diminution de succion due à un remouillage intervenant soit lors de la construction d'un ouvrage en terre, soit lors de sa mise en eau s'il s'agit d'un barrage, peut engendrer des déformations de distorsion et des redistributions de contrainte non négligeables.

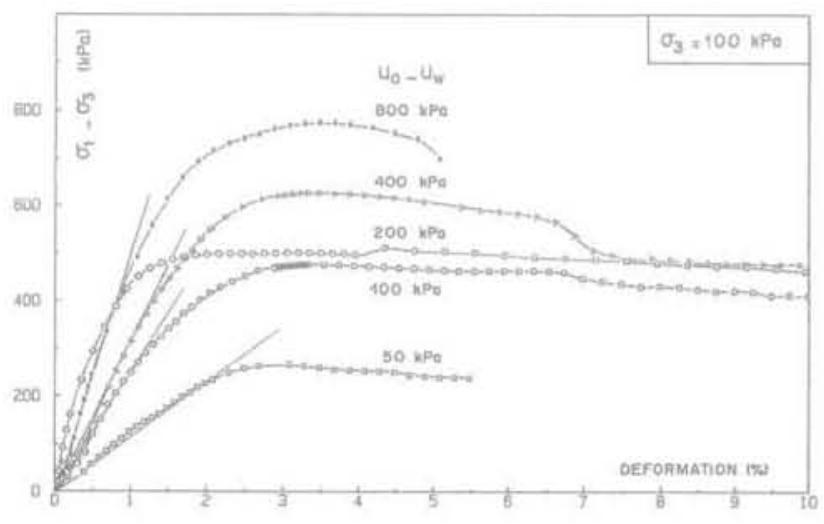

FG. 16 Essais triaxiaux à succion contrôlée sur un limon compacté (limon de Jossigny, Delage et al., 1987).

Suction controlled triaxial shear tests on a compacted silt (Jossigny silt, Delage et al., 1987).

L'incidence de la succion sur la cohésion et l'angle de frottement est moins claire et semble dépendre du type de sol considéré, comme le montre la figure 17 (Delage et Graham, 1995) qui regroupe des résultats obtenus sur divers sols ; Loess compacté de Trois Rivières (Maâtouk et al., 1995, triangles retournés), argiles de Guadalix et de Madrid (Escario et Saez, 1986, carrés et triangles respectivement), et limon de Jossigny (Delage et al., 1987, losanges). Sur la figure, les points représentatifs de l'angle de frottement (axe des ordonnées de gauche) sont en noir, et ceux représentant la cohésion (axe des ordonnées de droite) en $\alpha$ blanc $»$. Dans tous les cas, une succion croissante engendre une augmentation de la résistance mécanique du sol, qui se fait toujours par augmentation de la cohésion à partir de valeurs proches de zéro; l'observation de la figure montre que cette augmentation est analogue pour les deux argiles et le limon : une succion de $100 \mathrm{kPa}$ donne une cohésion de l'ordre de $100 \mathrm{kPa}$, et une succion de $600 \mathrm{kPa}$ donne une cohésion de l'ordre de $200 \mathrm{kPa}$. L'augmentation de cohésion est bien plus forte pour le loess de Trois Rivières. Cette augmentation peut s'interpréter comme le renforcement de liens de cimentation entre les particules ou les agrégats de particules ; dans les sols fins, ce lien interagrégats est constitué d'argile hydratée qui colle les agrégats entre eux, est dont la force augmente avec la succion. 
En revanche, l'angle de frottement peut croître légèrement, comme dans le cas de l'argile grise de Guadalix, être sensiblement constant (argile de Madrid), ou décroitre (limon de Jossigny et loess de Trois Rivières). Pour un même sol, l'observation des photos de la figure 5 semble confirmer que des réponses foncièrement différentes puissent être obtenues avec des caractéristiques de compactage différentes.

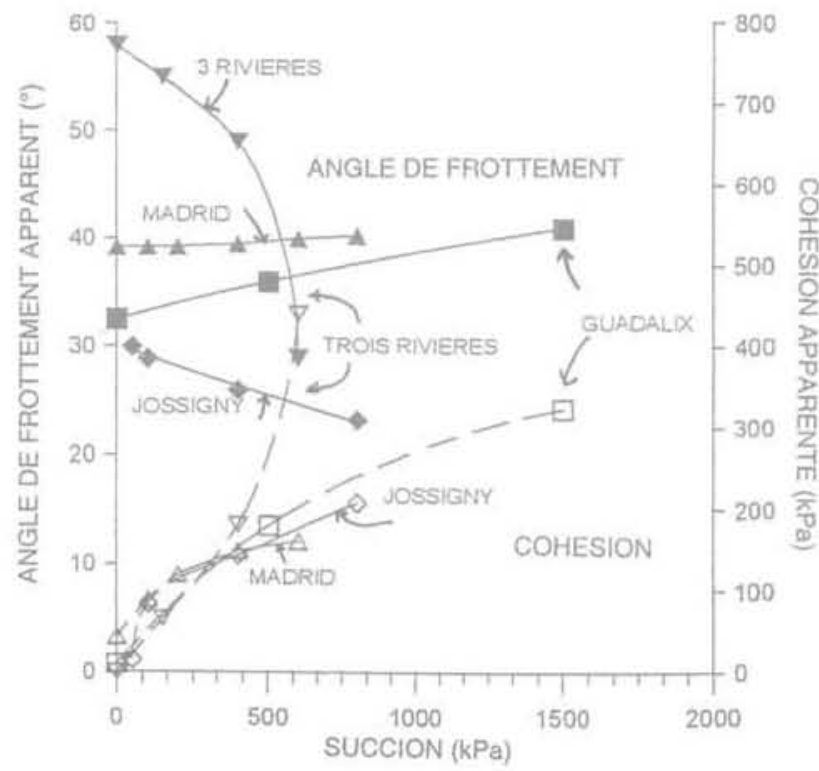

FIG. 17 Propriétés de résistance au cisaillement de divers sols (d'après Delage et Graham. 1995).

Shear strength properties of various soils (after Delage et Graharn, 1995).

\section{Conclusion}

Un certain nombre de concepts de la mécanique des sols non saturés ont été développés pour permettre une meilleure compréhension des phénomènes intervenant dans le comportement des sols compactés, et donc des ouvrages en remblai. Ce lien avec la mécanique des sols non saturés est assez rarement fait dans la pratique, alors qu'il facilite la compréhension du fonctionnement des ouvrages en terre. Le paramètre important pris en compte est la succion de l'eau interstitielle et ses variations, qu'on associe au diagramme de compactage. La microstructure des sols compactés est décrite, et les grands traits déjà observés sur d'autres sols sont vérifiés pour le limon de Jossigny, avec une structure en agrégats du côté sec, et une structure matricielle du côté humide. Les réponses en compression des sols compactés sous diverses conditions sont décrites, et permettent d'aboutir à la notion de surface d'état, qui constitue une première modélisation assez simple du comportement volumique des sols compactés sous des variations décroissantes du degré de saturation. En termes de résistance au cisaillement, les quelques données expérimentales actuellement disponibles montrent que le sol se renforce quand la succion augmente et qu'il sèche, par une augmentation de cohésion. En revanche, le sens des variations de l'angle de frottement varie selon la nature du sol, et probablement ses paramètres de compactage, densité et teneur en eau. Ici, des investigations expérimentales sont nécessaires pour éclairer davantage un point finalement encore assez mal connu. Les traits de comportement décrits dans cet article permettent d'aboutir à une modélisation simplifiée du comportement des ouvrages en remblai, qui sera abordée dans une prochaine publication.

\section{Bibliographie}

Alonso E.E., Gens A...Josa A. - $\alpha$ A constitutive model for partially saturated solis 1 . Géotechnique 40, 1990, p. 405-430.

Alonso E.E. Battle F. Gens A. Lloret A. "Consolidation analysis of partially saturated soils. Apnlication to earth. dams construction 3. Proceedings of the International Conference on Numerical Methods in Geotechnics, Innsbruck, vol. 3. 1988, p. 1303-1308.

Auvinet $G$. Espinoza I - $-\alpha$ impermeabilization of a 300 hectares cooling pond $\mathrm{x}$. Proceedings, Symposium on permeability and groundwater contaminant transport, Philadelphia ASTM, Special Technical Publication 746, 1979, p. 151-167.

Benson C.. Daniel D. - is Influence of clods on hydraulic conductivity of compacted clays n. Journal of Geotechnical Engineering, 116 (8), 1990, p. 1231-1248.

Bishop A.W. - Teknisk Ukeblad n 39, 1959, p. 859-863.

Barden L.. Madedor A.O. Sides G.R. "Volume change characteristics of unsaturated clays n, ASCE lournal of the Soil Mechanics and Foundation Division, vol. 95 (SM1), 1969, p. 35-52.

Black W.P.M. - « A method for estimating the California Bearing Ratio of cohesive soils from plasticity data in. Géotechnique 12, 1962, p. 271-282.
Cabot L., Le Bihan J.-P - " Ouelques propriétés d'une argile sur la "ligne optimale de compactage n. Revue Canadienne de Géotechnique vol. 30, 1993 p. 1033-1040.

Coleman J.D. - $\alpha$ Stress-strain relations for partly saturated soils $m$. Correspondence to Géotechnique 12. 1962, p. 348-350.

Cui Y.J., Delage, P, - a Yielding and plastic behaviour of an unsaturated compacted silt ». Géotechnique 46 (2), 1996, p. 291-311.

Delage P., Cui Y.J. - «L'eau dans les sols non saturés 3. Techniques de 1'Ingénieur, coll. "Construction $n$, article C301, 2000

Delage P., Cui Y.J. - «Comporternent mécanique des sols non saturés v. Technique de I'Ingénieur, coll. $\approx$ Construction $»$. article C 302, 2001.

Delage P.. Lefebvre G. - $\alpha$ Study of the structure of a sensitive Champlain clay and of its evolution during consolidation s. Canadian Geotechnical Journa! 21. 1984, p. 21-35.

Delage P. Graham J. - - The mechanical behaviour of unsaturated soils $x$. Proceedings of the 1st International Conference on Unsaturated soils, vol. 3. Paris, Alonso and Delage eds. Balkema Presses des Ponts et Chaussẻes, 1995. p. 1223-1256.
Delage P., Suraj De Silva G.P.R. \& Influence de la succion sur la compres. sibilité d'un limon non saturé p. XIIf Conférence internationale de mécanique des sols et des travaux de fondations, New Delhi, vol. 1. 1994, D. 89-92

Delage P.. Howat M., Cui Y.J. - \& The relationship between suction and swelling properties in a heavily compacted unsaturated clay w, Engineering Geology 1-2 (50), 1998 , p. $31-48$

Delage P. Suraj de Silva G.P.R. De Laure E. - " Un nouvel appareil triaxial pour les sols non saturés $)$. Comptes rendus de la ge Conférence européenne de mécanique des sols et des travaux de fondations, vol. 1, 1987, p. 26-28.

Delage P. Audiguier M., Cui Y.J., Howat M. - « Microstructure of a compacted silty clay v. Canadian Geotechnical Journal 33, 1996, p. 150-158.

Escario V. - « Formulaciones para la definición de la resistencia a esfuerzo cortante de los suelos parcialmente satura. dos n, Boll. Soc. Esp. Mec. Suejos y Cim. 92, 1988, p. 3-12.

Escario V., Saez J. - - The shear strength of partly saturated soils ». Géotechnique 36 1986, p. 453-456.

Fredlund D.G., Morgenstern N.R. - «Constitutive relations for volume change in 
unsaturated soils s, Canadian Geotechnical Journal 13, 1977, p. 261-276.

Garcia-Bengochea I. Lovell C.W.. Altschaeffl A.G. - "Relation between pore size distribution and permeability of silty clay n, Journal of the Geotechnical Engineerting Division, ASCE, vol. 105, $n^{\circ}$ GT7. 1979, p. 839-856.

Gens A. - "Constitutive modelling : application to compacted soils v. Proceedings of the 1 st International Conference on Iincaturated Snits LINSAT 95. vol. 3. Balkema/Presses des Ponts et Chaussées, 1995, p. 1179-1200

Gens A. Alonso E.E., Suriol J., Lloret A. $\alpha$ Effect of structure on the volumetric behaviour of a compacted soil $\mathrm{x}$. Proceedings of the 1st International Conference on Unsaturated Soils UNSATY5, vol. 1. Baikema/Presses des Ponts, 1995, p. 8388.

Jennings J.E., Knight K. * The additional settlement of foundations due to collapse of sandy soils on wetting v. Proceedings of the 4th International Conference on soil Mechanics and Foundation Engineering, vol. 1, 1957, p. 316-319.

Juang C.H., Holtz R.D.- - Fabric, pore size distribution and permeability of sandy solls 1. Journal of Geotechnical Engineering, ASCE, vol. 112, n 9, 1987, p. 855-868.

Kassiff G., Ben Shalom A. - « Experimental relationship between swell pressure and suction 3, Geolechnique 21, 1971 p. 245-255.

Kouassi P. Breysse D., Girard H., Poulain D - a A new technique of kneading compaction in the laboratory 3. ASTM Geotechnical Testing Journal, vol. 23 (1). 2000, p. $72-82$.

Li Z.M. - a Compressibility and collapsibility of compacted unsaturated loessia. soils 11. Proceedings of the 1st International Conference on Unsaturated Soils UNSAT'95, vol. 1. Balkema/Presses des Ponts et Chaussées, 1995, p. 139-144.

Lambe T.W. - \& The structure of compacted clay n. ASCE J. Soil Mech. Found. Div. 84, 1958a, p. 1-34.

Lambe T.W. - $c$ The engineering behavior of compacted clay b. ASCE J. Soil Mech Found. Div. 84, 1958b, p. 1-35.

Maâtouk A., Leroueil S., La Rochelle P. "Yielding and critical state of a collapsible unsaturated silty soil 10. Géotechmique 45 (3), 1995, p. 465-477.

Matyas E.L., Rachakrishna H.S. - "Volume change characteristics of partially saturated soil 1. Géotechnique 18, 1968, p. $432-448$.

Mieussens C. - « Déformations cycliques ef irréversibles dans les remblais argileux x. Revue française de Géotechnique 90,2000 , p. 47-59.

Mitchell J.K. - Fundamentals of soil beha- viour. New York, John Wiley and Sons Inc, 1993.

Murayama S., Michiïro K., Sakaagami T, "Creep characteristics of sands \%. Soils and Foundations vol. 24, 1984, p. 1-15.

Nanda A., Delage P., Nedjat N., Fry J.J. Gatmiri B. - « Evaluation de la sécurité des rembiais en cours de construction : le code UDAM n. Revue francaise de Geotechnique 62, 1993, p. 23-34.

Pellerin F.M. - $\alpha$ La porosimétrie au mercure appliquée à l'étude géotechnique des sols et des roches m. Bulletin de llaison des laboratoires des ponts et chaussées vol. 106, 1979, p. 105-116.

Richards L.A. - is A pressure : membrane extraction apparatus for soil solution $n$. Soil Science vol. 51, 1941, p. 377-386.

a Statement of the Review Panel p. Proceedings of the Symposium on Moisture Equilibria and Moisture Changes in Soils Beneath Covered Areas, 1965, p. 7-21.

Sridharan A., Altschaeffl A.G. Diamond, S. - a Pore size distribution studies $n$. ASCE J. Soil Mech. Found, Div. 97, 1971. p. $771-787$.

Van Olphen $\mathrm{H}$ - An introduction to clay colloid chernistry. New York, John Wiley and Sons Inc (2nd ed), 1977.

Yoshimi Y., Osterberg J.O. - « Compression of partially saturated cohesive soilsn. ASCE J. of the Soil Mechanics and Foundations, Div, 89 (4) 1963, p. 1-24.

\section{Annexe : contrainte de Bishop}

L'expression de la contrainte " effective n de Bishop est la suivante :

$$
\sigma^{\prime}\left(\sigma-u_{a}\right)+\chi\left(u_{a}-u_{w}\right)
$$

$\chi$ étant un paramètre compris entre 0 et 1 , égal à 0 pour un sol sec (on a alors $\sigma^{\prime}=\left(\sigma-u_{a}\right)$ ) et à 1 pour un sol saturé (on a alors $\left.\sigma^{\prime}=\left(\sigma-u_{i}\right)\right)$. On voit que lors du remouillage d'un sol non gonflant (succion décroissante), $\sigma^{\prime}$ décroît car $\chi$ est positif. Si le concept était valide, le sol devrait gonfler, or il diminue de volume du fait de l'effondrement (Jennings et Burland 1962). 